NASA/TM-2001-211213

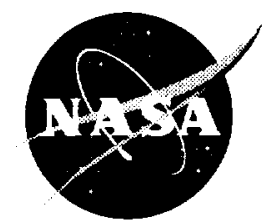

\title{
CSMA Versus Prioritized CSMA for Air-Traffic-Control Improvement
}

Daryl C. Robinson

Glenn Research Center, Cleveland, Ohio

Prepared for the

2002 Aerospace Conference

sponsored by the Institute of Electrical and Electronics Engineers

Big Sky, Montana, March 9-16, 2002

National Aeronautics and

Space Administration

Glenn Research Center 


\section{Acknowledgments}

I acknowledge Mr. Robert Kerczewski for recruiting me for this area of research and for funding it.

I also acknowledge the efforts of my summer interns, Proloy Bhattacharyya and Steve Drescher. Proloy built the network used in these simulations according to my specifications. Steve researched and generated, according to my specifications, all of the aircraft trajectories used in the simulations. They wrote process models and $C$ programs to validate their very good work, thereby allowing me to concentrate on writing all of the process models used in the simulation.

This report contains preliminary

findings, subject to revision as analysis proceeds.

Available from

NASA Center for Aerospace Information

National Technical Information Service 


\title{
CSMA Versus Prioritized CSMA for Air-Traffic-Control Improvement
}

\author{
Daryl C. Robinson \\ National Aeronautics and Space Administration \\ Glenn Research Center \\ 21000 Brookpark Road \\ Cleveland. Ohio 44135
}

\begin{abstract}
OPNET version 7.0 simulations are presented involving an important application of the Aeronautical Telecommunications Network (ATN), Controller Pilot Data Link Communications (CPDLC) over the Very High Frequency Data Link Mode 2 (VDL-2). Communication is modeled for essentially all incoming and outgoing nonstop air-traffic for just three United States cities: Cleveland. Cincinnati, and Detroit. There are 32 airports in the simulation, 29 of which are either sources or destinations for the air-traffic of the aforementioned three airports. The simulation involves 111 Air Traffic Control (ATC) ground stations, and 1,235 equally equipped aircraft-taking off, flying realistic free-flight trajectories, and landing in a 24-hr period. Collisionless. Prioritized Carrier Sense Multiple Access (CSMA) is successfully tested and compared with the traditional CSMA typically associated with VDL-2. The performance measures include latency, throughput. and packet loss. As expected. Prioritized CSMA is much quicker and more efficient than traditional CSMA. These simulation results show the potency of Prioritized CSMA for implementing low latency, high throughput and efficient connectivity.
\end{abstract}

\section{INTRODUCTION}

Due to a lack of surveillance and communications coverage, in many parts of the world, aircraft are forced to fly routes and maintain separations that are inefficient from both a fuel and scheduling perspective. The total loss to airlines due to these inefficiencies is measured in billions of dollars [1]. The problem is expected to rapidly mushroom given the expected user demand for scheduled air service. The Advanced Air Transportation Technologies (AATT) Program has been instituted to develop new technologies that enable free-flight. an operating system in which pilots have the freedom to select their path and speed in real-time [2].

To implement free-flight. CPDLC is viewed as very important for the new aeronautical communications infrastructure. CPDLC will eliminate voice-only communications.
In the simulations of this paper, realistic ground-to-air and air-to-ground communications are achieved by assuming an effective, intact terrestrial network and by treating planes as traffic generators and sinks, in a manner analogous to the transparent usage of a traffic injector or "sniffer" in a network. Further, the idea of Prioritized CSMA is reintroduced and successfully tested through simulation. Prioritized CSMA trades off the use of an additional radio frequency in order to implement efficient CSMA without collisions. The benefit gained of efficient. collision-less CSMA is that the inefficiencies introduced by wasted time division multiple access (TDMA) time slots may be avoided.

\section{Simulation Focus}

The primary focus of the simulations is to examine the behavior of ATC communications over VDL- 2 in an aviation scenario involving a substantial amount of air and communications traffic. Both weather and terrain were ignored, and the simulation assumes a spherical earth. Indirect communication is not implemented in this "OPNET" (network simulation software tool) simulation so two nodes may communicate only when they are in direct line-of-sight. So extending the range of ground stations by bouncing signals off of the ionosphere is not permitted here. All incoming and outgoing nonstop air traffic for three cities was simulated. Given the time constraints for this research and the scope of this simulation, it was not desirable to simulate the communications architecture for the entire OSI stack. Since the media access control layer (MAC) layer is especially important in broadcast media, largely determining the limit of performance, heavy emphasis was placed upon the data link layer. VDL-2. So these simulations do not model the presentation, session. transport. or network layers, as it was of most interest to simulate the VDL-2 data link layer, which is being deployed. Perhaps the most important use of these simulations is to test Prioritized CSMA. 


\section{Simulation Overview}

As previously stated, the simulation involves 1,235 flights, 111 ATC transceivers or ground stations, and 32 airports. The take off, arrival, and flight times for one day were based on real flight plans obtained from the airports. Instead of actually modeling the fact that one plane may make several flights, a separate OPNET mobile airplane node is used for each flight. For reasons discussed later, CPDLC messages in these simulations have a 5,000 bit mean file size. CPDLC file sizes are chosen according to the normal distribution. CPDLC messages have a variance of 2500 . They have a mean interarrival time of $6 \mathrm{~min}$. using the exponential distribution. All CPDLC transceivers operate at $136 \mathrm{MHz}$ with a $10 \mathrm{KHz}$ bandwidth.
Message Length

Although 5,000 bit message lengths are somewhat excessive for CPDLC messages, they were chosen so that the effects of message collisions could be better studied given the lower amount of aeronautical communications traffic present in these presently bounded simulations.

\section{Ground Stations}

It was not intended to perfectly replicate the National Aerospace System (NAS) in these simulations, but to provide a data communications environment in the simulation similar to that in the NAS. Consequently we did not require an exact distribution of ground stations. Instead, for research purposes.

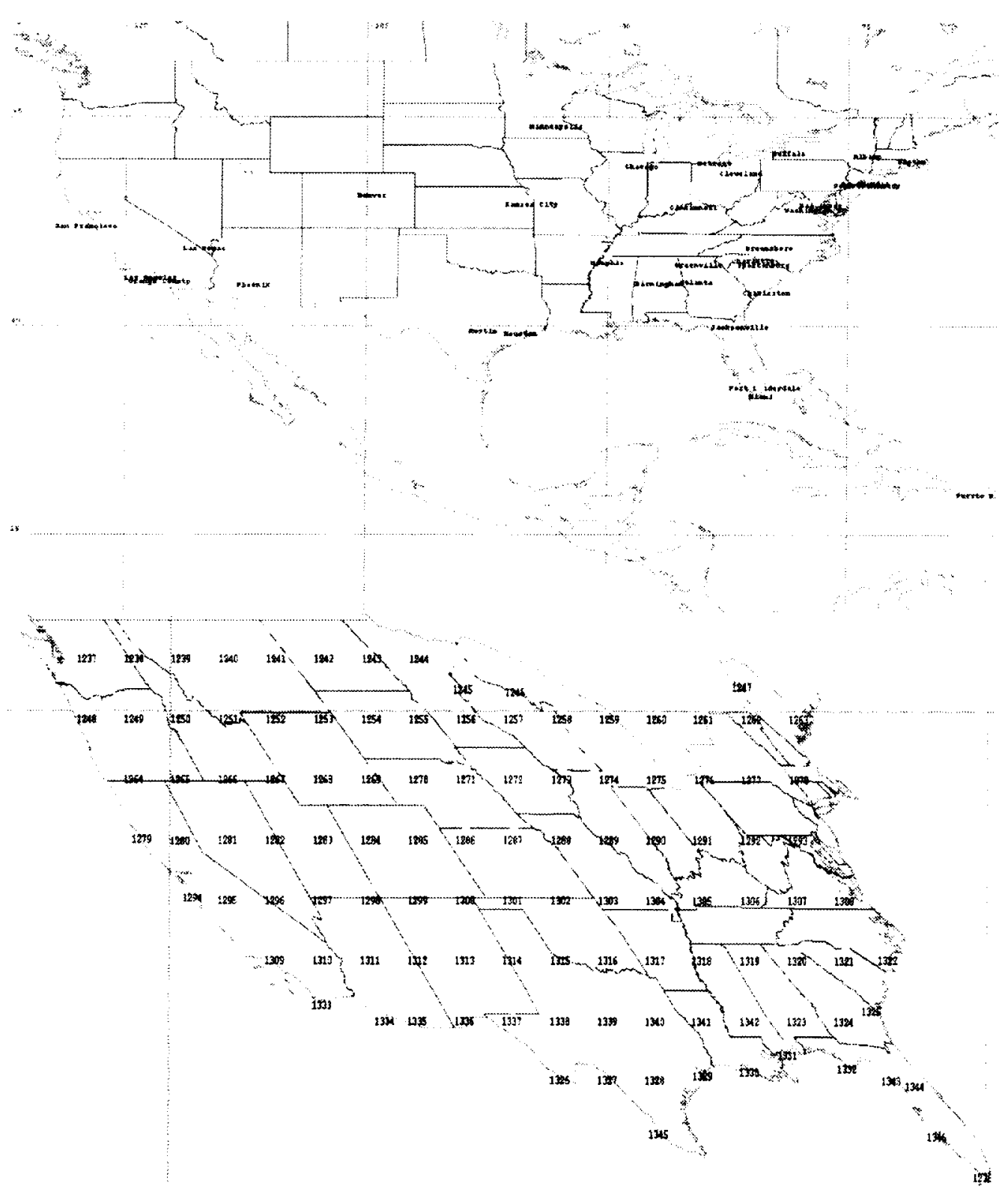

Figure 1: 32 airports (top) and 111 ground stations. 


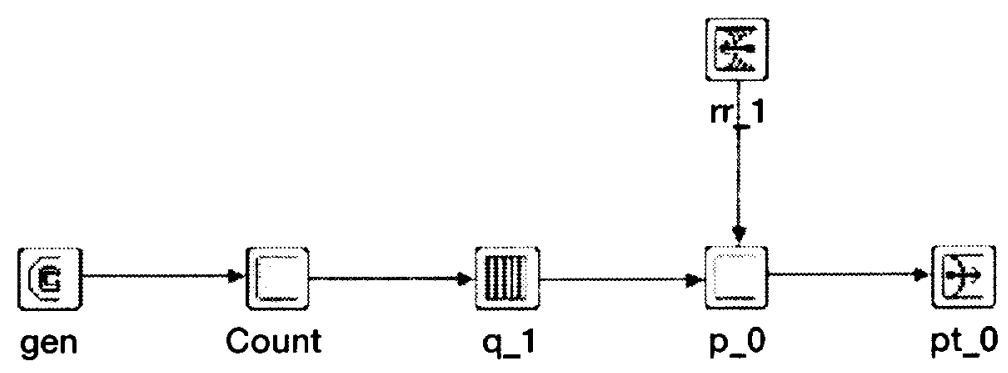

Figure 2: CPDLC node architecture.

we distributed them uniformly throughout the United States. A $100 \mathrm{~m}$ ground station may maintain direct line-of-sight communication with an airplane having an average altitude of $3.43 \mathrm{mi}$. for about $300 \mathrm{~km}$. So we used an average spacing of $290 \mathrm{~km}$ between adjacent ground stations. According to our calculations, this spacing should be sufficient to ensure continuous air to ground and ground to air communications. The ATC tower at Hopkins is $199 \mathrm{ft}=60.93 \mathrm{~m}$ in height. The simulation approximates the altitude of typical VDL ground stations as half that value. $30.47 \mathrm{~m}$. There are 111 ground stations in the simulation. Additionally, there is an air traffic control tower at each of the 32 airports. Figure 1 shows a view of the 32 airports and 111 ground stations involved in the simulation.

The ground stations are capable of detecting the presence of a plane and only send CPDLC messages if there is a plane within its $290 \mathrm{~km}$ airspace to receive them. Due to the functioning of Prioritized CSMA, the ground stations are coordinated and produce no uplink interference.

\section{Details}

Each airport is initially stocked with many planes, which will take off for one of the remaining 31 airports during the course of the $24 \mathrm{hr}$ simulation. Again. all simulated flights are nonstop. Each ground station. including air traffic control towers. consists of a CPDLC transceiver. Each airplane has identical communications architecture. CPDLC exists only between aircraft and ground stations. The CPDLC transmission node architecture is shown in Fig. 2.

In Fig. 2, "gen" is a clocked generator of packets. " $q_{-} l$ " is a queue to buffer the packets. "p_0" is a processor module, which decides whether to leave the packets in the queue or to forward them on to the radio transmitter through pt_o.

Airline officials provided us with typical flight altitudes as a function of distance traveled for various ranges. A typical plot of a trajectory profile is shown in Fig. 3.

Cruise altitudes used in the simulation depend on the range of the flight. The histogram, in Fig. 4. of the number of planes in flight, as a function of simulation time in minutes is based on the actual data from the airports and is not an output of simulation. This histogram can be used to understand traffic loading in the simulation. Air traffic begins $1 \mathrm{hr} 10 \mathrm{~min}$ into the sinulation and continues throughout the $24 \mathrm{hr}$ simulation.

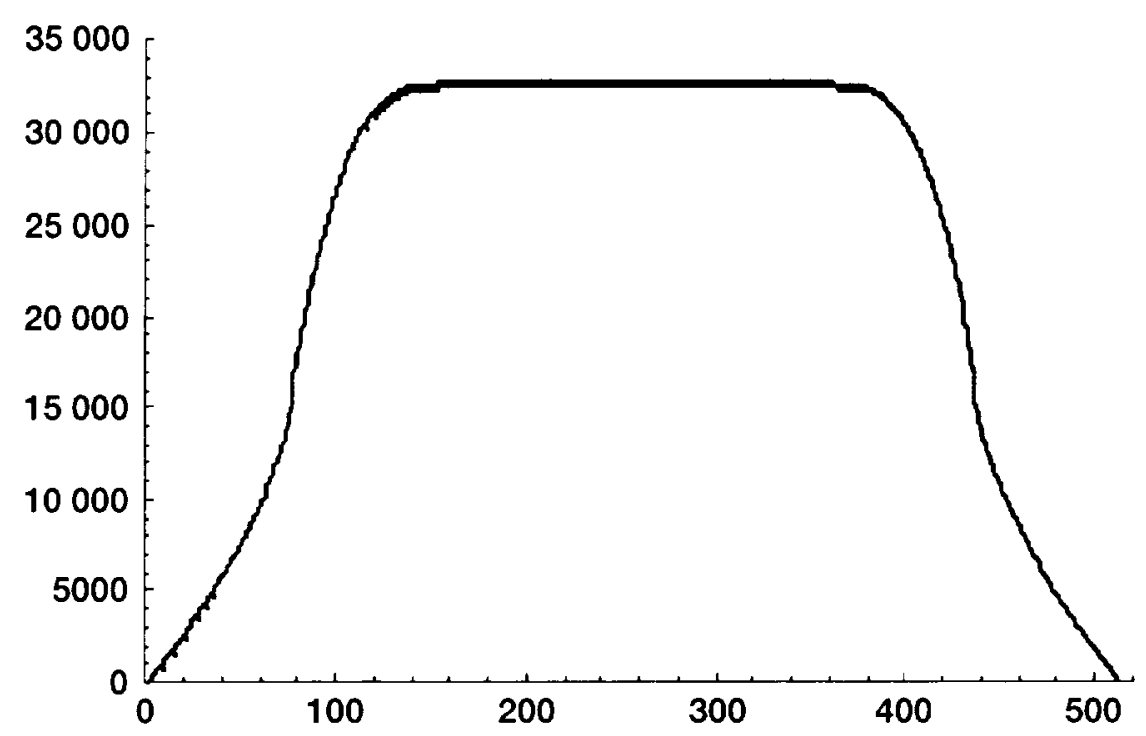

Figure 3: Flight trajectory profile: Cleveland to Albany. Altitude ( $\mathrm{ft}$ ) vs. time ( $\mathrm{min}$ ). 


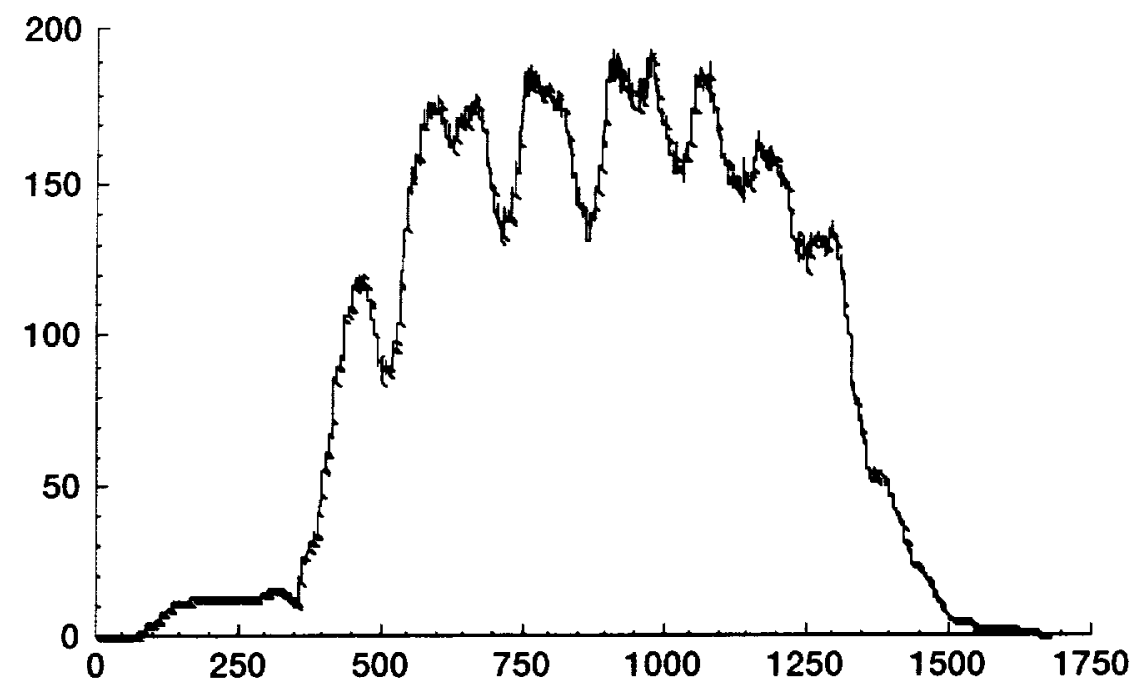

Figure 4: Number of planes aloft vs. time (min).

From the airport data, the average number of planes flying is 90.8. So the expected number of CPDLC messages originating from aircraft is $90.8(1,440 / 6) \approx 21,792$, where 1,440 is the number of minutes in a day. Additionally, each of these 90.8 aircraft is in view of approximately 2.25 unique ground stations, bringing the total number of expected CPDLC messages to $2.25(21,792)=49.032$, which is almost exactly what is observed later in the presented results. The peak traffic is at $(60 \mathrm{~s} / \mathrm{min})(910 \mathrm{~min})=54,600 \mathrm{~s}$ or $3: 10 \mathrm{p} . \mathrm{m}$.

\section{CSMA Discussion}

A single communications frequency is used for radio frequency conservation. Just as in CB radio, one party communicates at a time. But as east coast truckers may talk to their east coast neighbors while west coast truckers may simultaneously talk to their west coast neighbors-on the same frequency as their east coast counterparts-without interference, so in the simulations here, different line-of-sight groups can communicate on the same frequency simultaneously without interference.

CSMA is contention-based. All parties listen to the channel. When the channel is free, many parties contend for it until after a random back-off time. Eventually, one party gains control of the channel for uninterrupted usage. Because of the contention process, collisions can be inefficient.

\section{Prioritzed CSMA}

In Prioritized CSMA, each communications party is assigned a priority for transmission, based on its need to transmit. In these simulations, transmission priority is effectively granted on a first come, first serve basis. Effectively, if the medium is busy, each transmitter receives a waiting ticket; when its number comes up, the transmitter takes its turn. When the channel is free, instead of a random back-off time elapsing before one node gains usage of the channel, in Prioritized CSMA, the node with the next higher priority begins uninterrupted transmission immediately in an orderly fashion, without contention. By choosing to study Prioritized CSMA, we simultaneously accomplish two purposes. We can test this new idea and also obtain the upper bound for performance of VDL-2 with the given traffic of the simulation. Because of its random backoff time, VDL-2 should not perform as well as Prioritized CSMA.

\section{Details}

It is assumed that in a real implementation of the idea of Prioritized CSMA, both planes and ground stations include a connection transmission (cnctrans) transmitter. Much like an Automatic Dependent Surveillance-Broadcast Mode (ADS-B) transmitter, this transmitter would broadcast cnctrans packets at regular intervals. The cnctrans packets are nearly length zero and contain the unique source identification code (srcid) of the transmitting node. They also contain a time stamp and the transmission time remaining, ending, or beginning of that node. When a node receives a cnctrans packet, it updates an array of cnctrans information from its neighbors. If a cnctrans packet has not been received from a node in $\Delta t$, it is assumed unreachable. When a node seizes the channel, all nodes wait until it is finished. Each node waits until the farthest neighbor of the last transmitting node has received the transmission. When the transmission is finished, the next node begins orderly transmission. The cnctrans packets do not collide since they are small and each node is assigned a unique phase lag with which to broadcast them. 


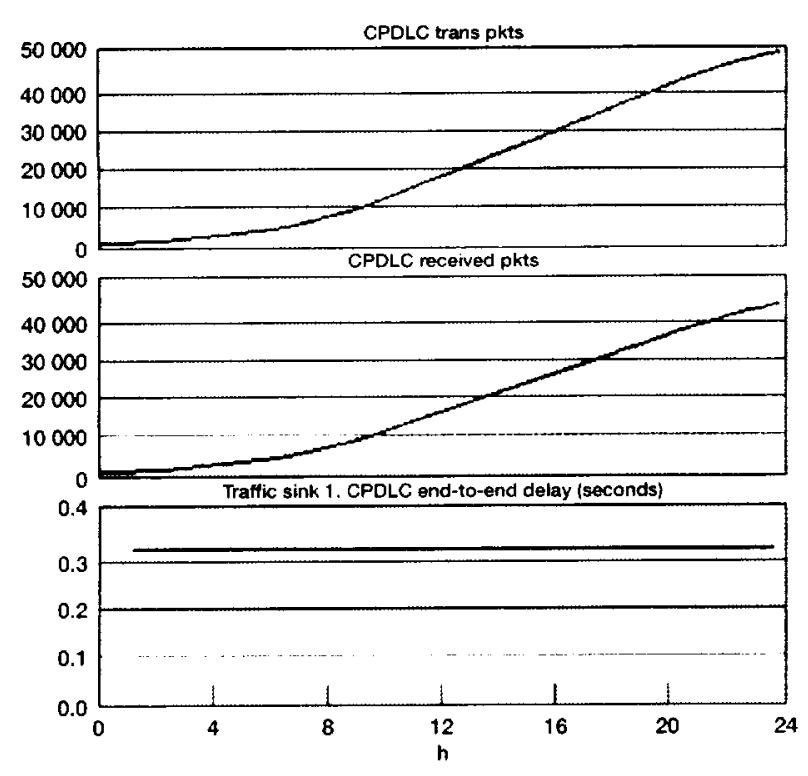

Figure 5: CPDLC packet reception and delay, I.

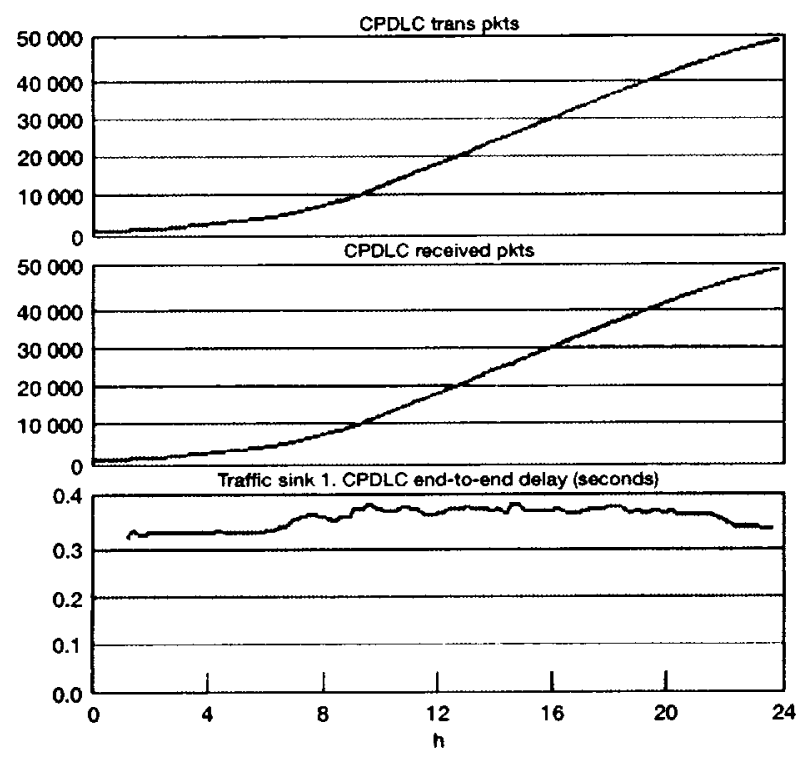

Figure 7: CPDLC packet reception and delay, III.

\section{Simulation Results and Analysis}

Results

There were four simulation runs, which used either no CSMA (no access scheme-transmit at will) or Prioritized CSMA, and which either used transceivers and queues with data rates and service rates of $31.5 \mathrm{Kbps}$ or $1.544 \mathrm{Mbps}$ :

I: no CSMA, $31.5 \mathrm{Kbps},[3]$

II: no CSMA, 1.544 Mbps

III: Prioritized CSMA, 31.5 Kbps.

IV: Prioritized CSMA, $1.544 \mathrm{Mbps}$

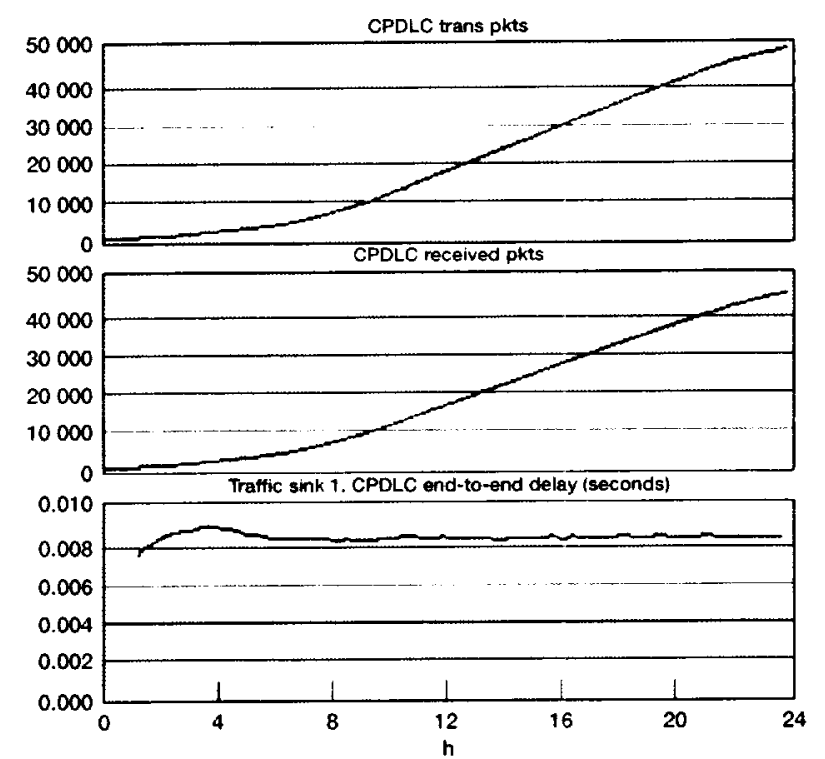

Figure 6: CPDLC packet reception and delay, II.

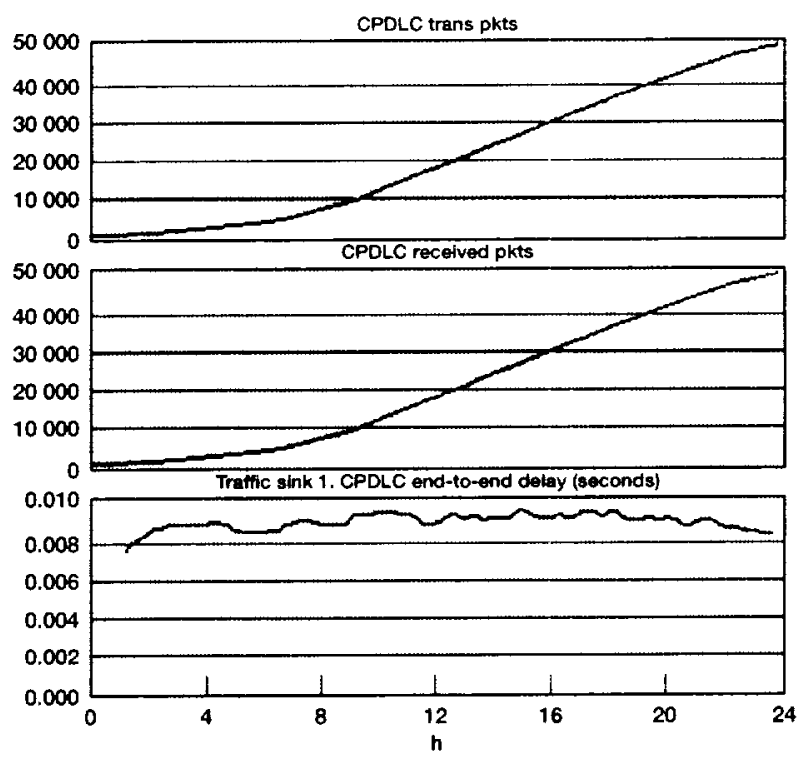

Figure 8: CPDLC packet reception and delay, IV.

The number of CPDLC messages received out of those transmitted for each of the Runs I to IV is respectively (43.673/49.904). (45,267/49,334), (49,926/49,931), and $(49,759 / 49,762)$. Plots of CPDLC transmitted and received packets for Runs I to IV are shown in Figs. 5 to 8. Included in those figures are plots of end-to-end delays for each run.

Only the runs using Prioritized CSMA successfully transmitted all CPDLC packets with negligible packet loss. These results show that this implementation of the idea of prioritized, collision-less CSMA works. Moreover, a comparison between the performance latencies in these simulations and the 95th percentile end-to-end delay requirement of $3 \mathrm{sec}$ [4] shows that Prioritized CSMA is remarkably quick and efficient. 
The numbers displayed above for Run III and IV show that there are between 3-5 packets lost. We have strong reason to believe that the loss is not due to a faulty implementation of Prioritized CSMA. We have determined that this is, instead, an error with the custom-built line-of-sight closure model that we used. We have observed in other runs that the packets that were lost. in both runs. did not reach any receiver in the entire network. These losses could be the result of our closure model determining that line-of-sight exists while OPNET's closure model determines otherwise. Nevertheless, we assert that our closure model is highly accurate, but slightly imperfect, and is inspired by OPNET code. We also note that these aberrant losses are rare.

\section{Conclusions}

One thing is obvious from a comparison of Runs I and II with III and IV: Prioritized CSMA works. Prioritized CSMA would serve the same purpose for aeronautical communications traffic as the traffic light does for automobile traffic-to prevent collisions.

In the event that it is critical to receive messages without many retransmissions or with minimum latency, Prioritized CSMA may be very useful. Acknowledgments and retransmissions increase the amount of traffic, increasing the number of collisions and worsening communications throughput.

Forecasts suggest that air traffic will triple over the next 20 years. Simulation studies have been performed that show that there is an upper limit to the number of aircraft that may be supported using VDL-2, i.e., traditional CSMA [4]. The limitation exists because of the inherent inefficiencies present in contentious, disorderly CSMA. Plans are underway to replace VDL-2 (which has barely been deployed) as the national aviation data link scheme with VDL-3, referred to as NEXCOM, based on time division multiple access (TDMA). This transition may be most expensive and somewhat sudden. However, small add-on modules could be manufactured to mate with existing VDL-2 radios to implement Prioritized CSMA, thereby extending the lifetime of VDL-2.
It appears as though this simulation method could be used to obtain an upper limit for the performance of CSMA or as justification for further research into the use of Prioritized CSMA. Plans are underway to improve this simulation and to use it as the basis for future research.

The simulation of communication was effected without the complexity involved in the aeronautical telecommunications network. It is desirable to identify communications systems that work and can be proven through simulation. Presently, there is not that much simulation research supporting the envisioned ATN. In this research, continuous communication was achieved in a realistic aviation scenario. It is difficult to even begin to convincingly do this for communications based on the ATN stack.

\section{REFERENCES}

[1] Final Report of RTCA Task Force 3 on Free Flight Implementation, October 26, 1995.

[2] Tekla S. Perry, "In Search of the Future of Air Traffic Control,” IEEE Spectrum, August 1997.

[3] Daryl C. Robinson, "Air Traffic Control Improvement Using Prioritized CSMA," IEEE Aerospace Conference. January 2000.

[4] Brian T. Hung, "Modeling and Simulation of an Aeronautical CSMA Subnetwork," OPNETWORK 2001 , August 2001.

Daryl C. Robinson received his $B S$ in Mathematics from Case Western Reserve University (CWRU), MSEE from Cleveland State University, and is pursuing his doctorate at CWRU. Currently he is pursuing research for the AATT program. He has expertise in mathematical modeling and simulation and in his nonwork hours enjoys reading and writing thoughtful literature.

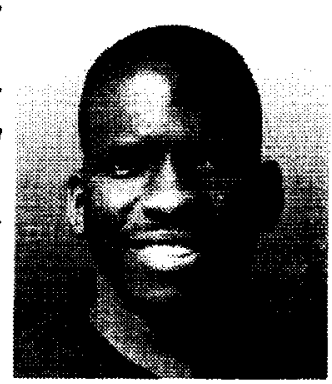




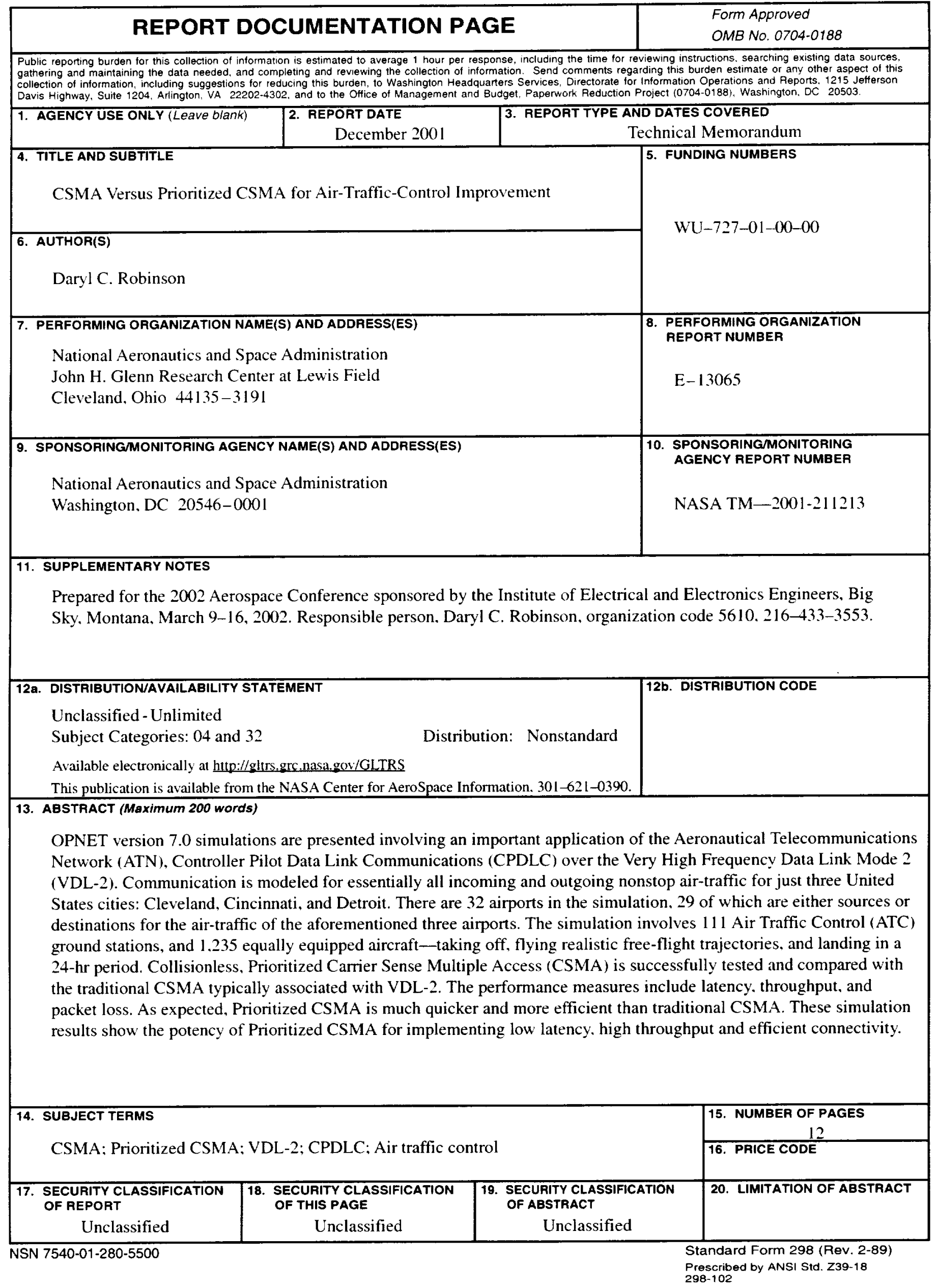


